\title{
A Novel Sliding-Mode Observer for Indirect Position Sensing of Switched Reluctance Motor Drives
}

\author{
Y. J. Zhan, Student Member, IEEE, C. C. Chan, Fellow, IEEE, and K. T. Chau, Member, IEEE
}

\begin{abstract}
A switched reluctance motor (SRM) drive generally requires a rotor position sensor for commutation and current control. However, the use of this position sensor increases both cost and size of the motor drive and causes limitations for industrial applications. In this paper, a novel indirect position sensing technique, namely, the sliding-mode observer, is proposed for SRM drives. The corresponding design approach and operating performance are provided to illustrate the fast convergence and high robustness of the observer against disturbances and variations.
\end{abstract}

Index Terms - Observers, position measurement, reluctance motor drives, variable-structure systems.

\section{INTRODUCTION}

G ENERALLY, a switched reluctance motor (SRM) drive requires an external or built-in rotor position sensor for commutation and current control. The position sensor, however, not only adds complexity, cost, and size to the whole drive system, but also causes limitation for industrial applications. Thus, in order to eliminate this physical position sensor, a number of indirect position sensing techniques have been proposed for SRM drives in recent years [1]-[9].

The proposed indirect position sensing techniques for SRM drives can be classified into two major methods. One of them is the so-called waveform detection method. Since the unsaturated phase inductance of an SRM varies significantly between the aligned and unaligned positions of the stator and rotor poles, this method is based on the measurement of inductance variation in one of the unenergized phases, as reported in [2], [6] and [8]. Although this sensorless operation can eliminate the position sensor, it suffers from the following drawbacks:

- generation of negative torque due to the use of a diagnostic pulse [6];

- limited resolution at high speeds due to the time delay caused by the previous diagnostic phase [2];

- added complexity due to the diagnostic circuitry.

Another key method is based on the fact that the terminal voltage and current of an SRM drive contain the necessary information to recast the rotor position. Thus, the Luenberger

Manuscript received November 18, 1997; revised September 10, 1998. Abstract published on the Internet January 18, 1999. This work was supported in part by the Hong Kong Research Grants Council and in part by the Committee of Research and Conference Grants of The University of Hong Kong.

The authors are with the Department of Electrical and Electronic Engineering, The University of Hong Kong, Hong Kong (e-mail: ktchau@eee.hku.hk).

Publisher Item Identifier S 0278-0046(99)02718-5. observer theory has been employed to estimate the rotor position angle of SRM drives by using the measured phase voltages and currents [1], [7], [9]. However, this linear observer has neglected the nonlinearity due to magnetic saturation and speed perturbation, and suffers from high sensitivity at low speeds due to the large feedback gain corresponding to a fixed pole assignment. As a result, the performance of this observer may not be fast enough for transient operation. Moreover, an accurate knowledge of both motor and load parameters is desired when using this observer.

In this paper, a novel position sliding-mode observer for SRM drives is proposed in which magnetic saturation, load disturbances, parameter variations, and model errors have been taken into account. Disturbance rejection capability and optimum convergence rate of initial estimation errors are quantitatively analyzed. This sliding-mode observer aims to offer the advantages of inherent robustness of parameter uncertainty and easy application to SRM drives. Due to the use of state reconstruction, this technique avoids the need of added diagnostic circuitry and the generation of negative torque. With the proposed technique, a sliding-mode observer model can successfully estimate the rotor position based on the measured motor voltages and the difference between the estimated and measured motor currents.

\section{MODELING OF SRM DRIVES}

By defining $\mathbf{V}=\left[V_{1}, \cdots, V_{n}\right]^{T}$ as the vector of terminal phase voltages, $\mathbf{i}=\left[i_{1}, \cdots, i_{n}\right]^{T}$ as the vector of terminal phase currents, $\Psi=\left[\psi_{1}, \cdots, \psi_{n}\right]^{T}$ as the vector of flux linkages, $\mathbf{H}(\theta)=\operatorname{diag}\left[H_{1}(\theta), \cdots, H_{n}(\theta)\right]$ as the matrix of phase reciprocal inductances, and $\mathbf{r}=\operatorname{diag}\left[r_{1}, \cdots, r_{n}\right]$ as the matrix of phase resistances, the system dynamics of SRM drives can be modeled as

$$
\left\{\begin{array}{l}
\frac{d \Psi}{d t}=-\mathbf{r} \mathbf{H}(\theta) \Psi+\mathbf{V}+\mathbf{w}_{\psi} \\
\frac{d \omega}{d t}=\frac{T_{e}-T_{l}}{J}-\frac{B}{J} \omega=-\frac{B}{J} \omega+\frac{T_{e}}{J}+w_{\omega} \\
\frac{d \theta}{d t}=\omega+w_{\theta} \\
\mathbf{i}=\mathbf{H}(\theta) \Psi \\
T_{e}=T_{e}(\mathbf{i}, \theta)
\end{array}\right.
$$

where $\mathbf{x}=(\Psi \omega \theta)^{T}$ is the state vector, $\mathbf{w}=\left(\mathbf{w}_{\psi}, w_{\omega}, w_{\theta}\right)^{T}$ is the disturbance matrix, $\omega$ is the rotor speed, $B$ is the damping coefficient, $J$ is the combined inertia of the rotor plus the mechanical load, $\theta$ is the rotor position, $T_{e}$ is the 


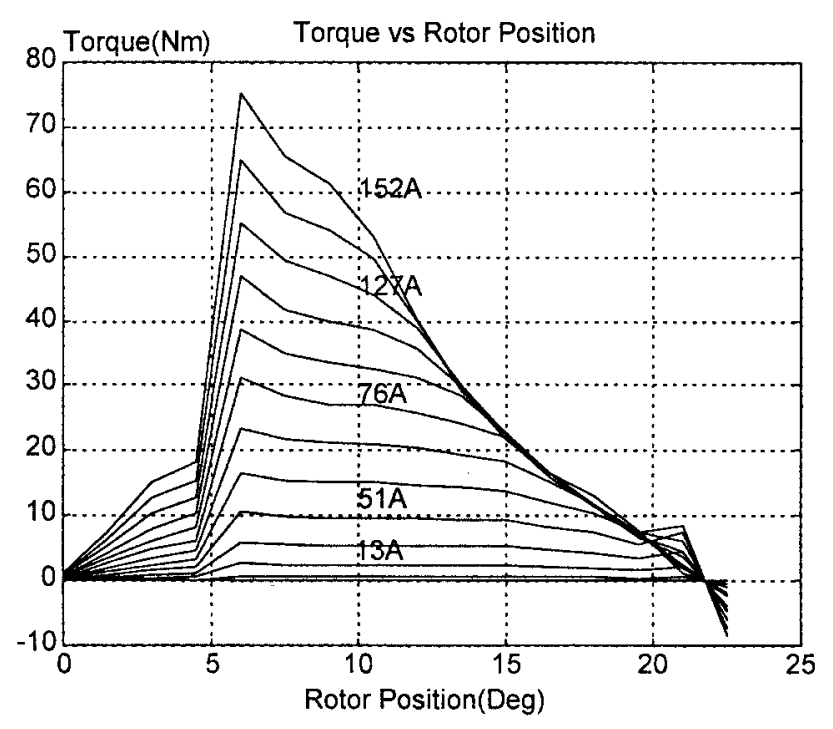

Fig. 1. Nonlinear characteristics of SRM.

electromagnetic torque, and $T_{l}$ is the load torque. Due to magnetic saturation, $T_{e}$ in (1) depends on both $\mathbf{i}$ and $\theta$. The corresponding nonlinear characteristics are shown in Fig. 1, which are obtained by using finite-element analysis during machine design. Moreover, the matrix $\mathbf{w}=\left(\mathbf{w}_{\psi}, w_{\omega}, w_{\theta}\right)^{T}$ consists of the model errors due to load disturbances and parameter variations. Although $T_{l}$ has been considered as a known value or even zero [7], this assumption may be invalid because of the fact that the load torque does exist and can hardly be measured precisely under inevitable speed perturbations. Therefore, in this paper, the load torque $T_{l}$ is treated as an external disturbance.

Based on the system experimental parameters of a typical three-phase 12/8-pole SRM drive shown in Table I, the reciprocal inductance of phase $a$ can be modeled by a nonlinear equation as given by :

$$
H_{a}(\theta)=1437+1134 \cos \theta+412 \cos (2 \theta) .
$$

Thus, the output current of phase $a$ can be expanded into a Taylor series at an equilibrium point $\mathbf{x}_{o}$, as shown in (3), at the bottom of the page. The output currents of phases $b$ and
TABLE I

System Parameters of SRM DRIVE.

\begin{tabular}{ll}
\hline Power & $4 \mathrm{~kW}$ \\
Phase & 3 \\
Stator poles & 12 \\
Rotor poles & 8 \\
DC voltage & $240 \mathrm{~V}$ \\
Rated current & $40 \mathrm{~A}$ \\
Rated torque & $12.8 \mathrm{Nm}$ \\
Stator resistance & $0.3 \Omega$ \\
Reciprocal inductance & $(1437+1134 \cos \theta) \mathrm{H}^{-1}$ \\
Viscous friction coefficient & $0.0012 \mathrm{Nms}^{2}$ \\
\hline
\end{tabular}

$c$ are similarly expanded. Hence, the output equation becomes (4), as shown at the bottom of the page, where

$$
\mathbf{H}_{\mathbf{0}}=\left(\begin{array}{ccc}
H_{a}\left(\theta_{o}\right) & 0 & 0 \\
0 & H_{b}\left(\theta_{o}\right) & 0 \\
0 & 0 & H_{b}\left(\theta_{o}\right)
\end{array}\right), \quad \mathbf{C}=\left(C_{a}, C_{b}, C_{c}\right)^{T}
$$

and

$$
\mathbf{D}_{\mathbf{0}}=\left(\begin{array}{c}
-1134 \sin \theta_{o} \psi_{a o} \\
-1134 \sin \left(\theta_{o}-120^{\circ}\right) \psi_{b o} \\
-1134 \sin \left(\theta_{o}+120^{\circ}\right) \psi_{c o}
\end{array}\right)
$$

are constant matrices.

\section{SLIDING-Mode ObSERVER OF SRM DRIVES}

\section{A. Sliding-Mode Observer and System Error Dynamics}

The sliding-mode observer theory [10] can be applied to the output equation given by (4). Thus, the sliding-mode observer is constructed as

$$
\left\{\begin{array}{l}
\frac{d \hat{\Psi}}{d t}=-\mathbf{r H}(\hat{\theta}) \hat{\Psi}+\mathbf{V}+\mathbf{K}_{\psi} \operatorname{sgn}(\hat{\mathbf{i}}-\mathbf{i}) \\
\frac{d \hat{\omega}}{d t}=\frac{\hat{T}_{e}}{J}-\frac{B}{J} \hat{\omega}+\mathbf{K}_{\omega} \operatorname{sgn}(\hat{\mathbf{i}}-\mathbf{i}) \\
\frac{d \hat{\theta}}{d t}=\hat{\omega}+\mathbf{K}_{\theta} \operatorname{sgn}(\hat{\mathbf{i}}-\mathbf{i}) \\
\hat{\mathbf{i}}=\mathbf{H}(\hat{\theta}) \hat{\Psi} \\
\hat{T}_{e}=\hat{T}_{e}(\mathbf{i}, \hat{\theta})
\end{array}\right.
$$

where ${ }^{\wedge}$ denotes the corresponding estimated value. In (5), the innovation terms are due to the error of the estimated

$$
\begin{aligned}
i_{a} & =\left.H_{a}(\theta) \psi_{a} \approx\left[\frac{\partial H_{a}(\theta) \psi_{a}}{\partial \psi_{a}}, \frac{\partial H_{a}(\theta) \psi_{a}}{\partial \psi_{b}}, \frac{\partial H_{a}(\theta) \psi_{a}}{\partial \psi_{c}}, \frac{\partial H_{a}(\theta) \psi_{a}}{\partial \omega}, \frac{\partial H_{a}(\theta) \psi_{a}}{\partial \theta}\right]\right|_{\mathbf{x}=\mathbf{x}_{o}}\left(\mathbf{x}-\mathbf{x}_{o}\right) \\
& =\left[H_{a}\left(\theta_{o}\right), 0,0,0,-1134 \sin \left(\theta_{o}\right) \psi_{a o}\right] \mathbf{x}+C_{a}
\end{aligned}
$$

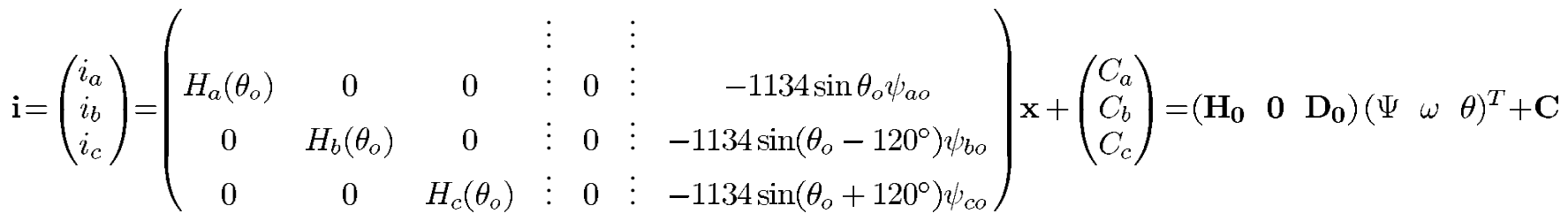

$$
\begin{aligned}
& =\left(\begin{array}{lll}
\mathbf{H}_{\mathbf{0}} & \mathbf{0} & \mathbf{D}_{\mathbf{0}}
\end{array}\right) \mathbf{x}+\mathbf{C}
\end{aligned}
$$




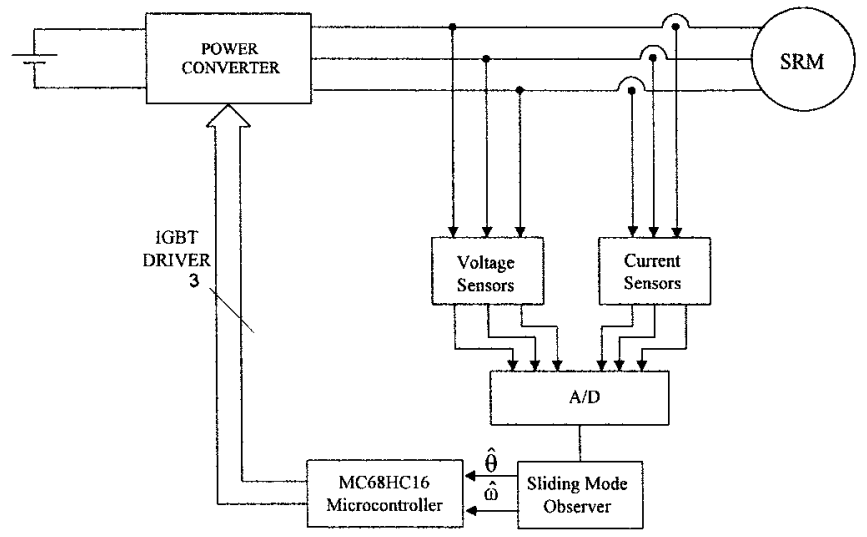

Fig. 2. System configuration of SRM drive.

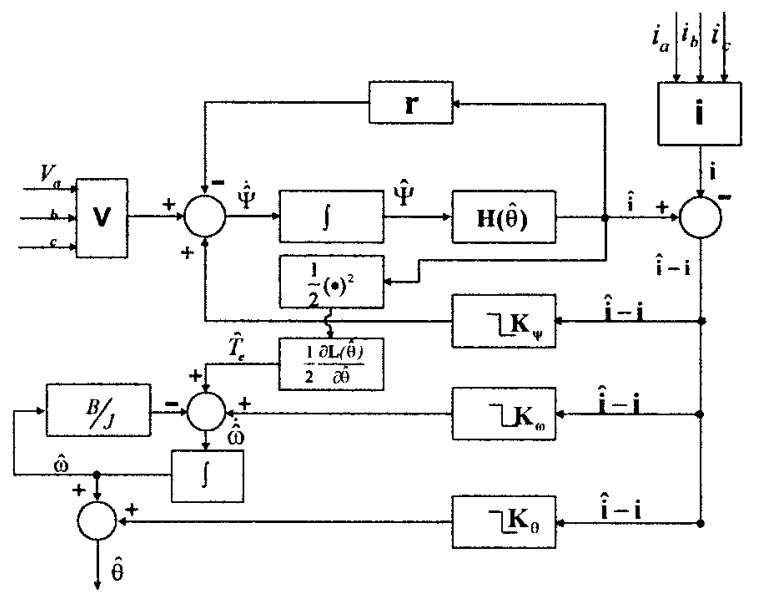

Fig. 3. Sliding-mode observer configuration.

and measured output currents multiplied by the switching gains $\mathbf{K}_{\psi}, \mathbf{K}_{\omega}$, and $\mathbf{K}_{\theta}$. It should be noted that $\mathbf{K}_{\psi}=$ $\operatorname{diag}\left(k_{\psi} \quad k_{\psi} k_{\psi}\right)$ is a matrix, whereas both $\mathbf{K}_{\omega}=k_{\omega}\left(\begin{array}{lll}1 & 1 & 1\end{array}\right)$ and $\mathbf{K}_{\theta}=k_{\theta}\left(\begin{array}{lll}1 & 1 & 1\end{array}\right)$ are the row vector gains. All of them are the essential parameters for ensuring the sliding mode of the observer. As shown in (5), the estimated electromagnetic torque $\hat{T}_{e}$ is calculated by using the measured phase currents $\mathbf{i}$, the estimated rotor position $\hat{\theta}$, and the nonlinear characteristics given by Fig. 1 .

Fig. 2 shows the system configuration of the proposed SRM drive using a sliding-mode observer. The corresponding configuration of the sliding-mode observer is illustrated in Fig. 3. The inputs of the sliding-mode observer are the source line-to-line voltages supplied by the PWM converter. The estimated three-phase currents $\hat{\mathbf{i}}=\left(\begin{array}{lll}\hat{i}_{a} & \hat{i}_{b} & \hat{i}_{c}\end{array}\right)^{T}$ are compared with the corresponding measured currents $\mathbf{i}=\left(\begin{array}{lll}i_{a} & i_{b} & i_{c}\end{array}\right)^{T}$ and the differences of these currents are fed back through the sign functions. The system errors of this observer are defined as

$$
\begin{aligned}
\mathbf{e}_{\psi} & =\hat{\Psi}-\Psi=\left(\begin{array}{lll}
\hat{\psi}_{a}-\psi_{a} & \hat{\psi}_{b}-\psi_{b} & \hat{\psi}_{c}-\psi_{c}
\end{array}\right)^{T} \\
e_{\omega} & =\hat{\omega}-\omega \\
e_{\theta} & =\hat{\theta}-\theta \\
\mathbf{s} & =\mathbf{e}_{i}=\hat{\mathbf{i}}-\mathbf{i}=\mathbf{c}(\hat{\mathbf{x}}-\mathbf{x}) .
\end{aligned}
$$

Substituting (1) and (5) into the derivatives of (6)-(8), the error dynamics can be obtained as

$$
\begin{aligned}
\frac{d \mathbf{e}_{\psi}}{d t} & =-\mathbf{r} \cdot \mathbf{e}_{i}+\mathbf{K}_{\psi} \operatorname{sgn} \mathbf{e}_{i}-\mathbf{w}_{\psi} \\
\frac{d e_{\omega}}{d t} & =-\frac{B}{J} e_{\omega}+\frac{1}{J}\left(\hat{T}_{e}-T_{e}\right)+\mathbf{K}_{\omega} \operatorname{sgn} \mathbf{e}_{i}-w_{\omega} \\
\frac{d e_{\theta}}{d t} & =e_{\theta}+\mathbf{K}_{\theta} \operatorname{sgn} \mathbf{e}_{i}+w_{\theta} .
\end{aligned}
$$

\section{B. Determination of Switching Gains $\mathbf{K}_{\psi}$ and $\mathbf{K}_{\theta}$}

To guarantee the system sliding mode, the sliding surface $\mathbf{s}$ on the state output $\mathbf{i}$ and its derivative $\dot{\mathbf{s}}$ are defined as

$$
\begin{aligned}
\mathbf{s} & =\hat{\mathbf{i}}-\mathbf{i}=\left(\begin{array}{lll}
\mathbf{H}_{0} & 0 & \mathbf{D}_{0}
\end{array}\right)\left(\begin{array}{lll}
\hat{\Psi}-\Psi & \hat{\omega}-\omega & \hat{\theta}-\theta
\end{array}\right)^{T} \\
& =\mathbf{H}_{0} \mathbf{e}_{\psi}+\mathbf{D}_{0} e_{\theta} \\
\dot{\mathbf{s}} & =\mathbf{H}_{0} \dot{\mathbf{e}}_{\psi}+\mathbf{D}_{0} \dot{e}_{\theta} .
\end{aligned}
$$

Hence, the sufficient condition of the sliding mode is expressed as

$$
\begin{aligned}
\mathbf{s}^{T} & \cdot \dot{\mathbf{s}} \\
& =\left(\mathbf{H}_{0} \mathbf{e}_{\psi}+\mathbf{C}_{0} e_{\theta}\right)^{T}\left(\mathbf{H}_{0} \dot{e}_{\psi}+\mathbf{C}_{0} \dot{e}_{\theta}\right) \\
& =\mathbf{e}_{\psi}^{T} \mathbf{H}_{0}^{T} \mathbf{H}_{0} \dot{\mathbf{e}}_{\psi}+e_{\theta} \mathbf{C}_{0}^{T} \mathbf{H}_{0} \dot{\mathbf{e}}_{\psi}+\mathbf{e}_{\psi}^{T} \mathbf{H}_{0}^{T} \mathbf{C}_{0} \dot{e}_{\theta}+e_{\theta} \mathbf{C}_{0}^{T} \mathbf{C}_{0} \dot{e}_{0} \\
& <0 .
\end{aligned}
$$

Inequality (15) constrains the trajectories pointing toward the surface $\mathbf{s}$, and is referred to as the sliding-mode condition. The idea behind this condition is to choose a well-behaved function of the tracking error $\mathbf{s}$, and then determine the switching gains $\mathbf{K}_{\psi}, \mathbf{K}_{\omega}$, and $\mathbf{K}_{\theta}$ to keep $\mathbf{s} \equiv \mathbf{0}$ and $\dot{\mathbf{s}} \equiv \mathbf{0}$ despite the presence of model imprecision and disturbances. Thus, this sliding observer possesses intriguing properties in the presence of measurement noise, as well as predictably robust properties in the presence of model errors.

Since $\mathbf{H}_{0}>\mathbf{0}$ and the sign of $\mathbf{D}_{0}$ depends on $\sin (\theta)$, the sliding-mode condition given by (15) can be further expressed as

$$
\begin{aligned}
\mathbf{e}_{\psi}^{T} \dot{\mathbf{e}}_{\psi} & <0 \\
e_{\theta} \dot{e}_{\theta} & <0 .
\end{aligned}
$$

Substitution of (6) and (10) into (16) yields

$$
(\hat{\Psi}-\Psi)^{T} \cdot\left(-\mathbf{r e}_{\mathbf{i}}+\mathbf{K}_{\psi} \operatorname{sgn}\left(\mathbf{e}_{\mathbf{i}}\right)-\mathbf{w}_{\psi}\right)<0 .
$$

In order to generate the sliding mode, the switching gain $\mathbf{K}_{\psi}$ should be chosen so as to be large enough to satisfy (18). Although it is usually claimed that the sliding mode generally yields robustness against disturbances, it should be noted that there is a restriction on this property. As to the sliding-mode observer, the sliding hyperplane is determined by the system itself, whereas the switching gain $\mathbf{K}_{\psi}$ can be arbitrarily assigned to attain robustness against disturbances. However, the restriction on the assignment of the switching gain $\mathbf{K}_{\psi}$ comes from the requirement that the observer is stable. If the gain is too large, a great amount of ripples may result, causing the estimation errors. Therefore, a tradeoff should be made between the robustness and the stability. Since 
$\mathbf{i} \propto \Psi$ and the bound of disturbance $\mathbf{w}_{\psi}$ is known, $\mathbf{K}_{\psi}$ can be deduced from (18) as

$$
\mathbf{K}_{\psi}<-\mathbf{r}\left\|\mathbf{e}_{i}\right\|-\left\|\mathbf{w}_{\psi}\right\|
$$

where $\|\cdot\|$ is the quadratic norm of vector space.

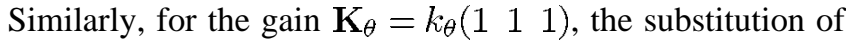
(8) and (12) into (17) yields

$$
(\hat{\theta}-\theta)\left(e_{\omega}+\mathbf{K}_{\theta} \operatorname{sgn}\left(\mathbf{e}_{i}\right)-w_{\theta}\right)<0 .
$$

From (20), if $\hat{\theta}-\theta>0$, then $e_{\omega}+\mathbf{K}_{\theta} \operatorname{sgn}\left(\mathbf{e}_{i}\right)-w_{\theta}<0$ is satisfied. Since $\mathbf{i} \propto \theta^{-1}$ is valid on a vicinity of the sliding plane, then $\operatorname{sgn}\left(\mathbf{e}_{i}\right)<0$ is valid. Therefore, $k_{\theta}$ can be deduced as

$$
k_{\theta}>e_{\omega}-w_{\theta} .
$$

On the contrary, if $\hat{\theta}-\theta<0$, then $e_{\omega}+\mathbf{K}_{\theta} \operatorname{sgn}\left(\mathbf{e}_{i}\right)-w_{\theta}>0$ and $\operatorname{sgn}\left(\mathbf{e}_{i}\right)>0$, which leads to

$$
k_{\theta}>-e_{\omega}+w_{\theta} \text {. }
$$

In order to satisfy both (21) and (22), gain $k_{\theta}$ can be expressed as a uniform ultimate inequality

$$
k_{\theta}>\left|\mathbf{e}_{\omega}\right|+\left|w_{\theta}\right| .
$$

It is obvious that the resulting $k_{\theta}$ satisfies the inequality given by (20).

\section{C. $H_{\infty}$ Optimization of Switching Gain $\mathbf{K}_{\omega}$}

Since $e_{\omega}$ does not appear in the inequality given by (15), this switching gain $\mathbf{K}_{\omega}$ cannot directly be determined from the sliding-mode condition. According to the sliding-mode theory, once the sliding mode occurs, an equivalent control becomes valid [10]. On a vicinity of the sliding surface, the equivalent control satisfies $\mathbf{s}=(\hat{\mathbf{i}}-\mathbf{i})=\mathbf{0}$ and $\dot{\mathbf{s}}=\mathbf{c}(\dot{\hat{\mathbf{x}}}-\dot{\mathbf{x}})=\mathbf{c} \dot{\mathbf{e}}_{x}=\mathbf{0}$, thus, $\dot{\mathbf{e}}_{\psi}=\mathbf{0}$. By substituting it into (10) and omitting the small voltage drop term, it results in

$$
\dot{\mathbf{e}}_{\psi}=\mathbf{K}_{\psi} \operatorname{sgn}(\hat{\mathbf{i}}-\mathbf{i})-\mathbf{w}_{\psi}=\mathbf{0} .
$$

Hence, the rearrangement of (24) yields

$$
\mathbf{K}_{\psi} \operatorname{sgn}(\hat{\mathbf{i}}-\mathbf{i})-\mathbf{w}_{\psi} \cdot
$$

By defining $\mathbf{K}_{\omega}=\mathbf{L}_{\omega} \mathbf{K}_{\psi}$ and substituting it into (11), it can be expressed as

$$
\dot{e}_{\omega}=-\frac{B}{J} e_{\omega}+\mathbf{L}_{\omega} \mathbf{K}_{\psi} \operatorname{sgn}(\hat{\mathbf{i}}-\mathbf{i})+\frac{1}{J}\left(\hat{T}_{e}-T_{e}\right)-w_{\omega} .
$$

By substituting (25) into (26), it results in

$$
\dot{e}_{\omega}=-\frac{B}{J} e_{\omega}+\mathbf{L}_{\omega} \mathbf{w}_{\Psi}+\frac{1}{J}\left(\hat{T}_{e}-T_{e}\right)-w_{\omega} .
$$

After defining the following parameter:

$$
w_{\omega}^{\prime}=\frac{1}{J}\left(\hat{T}_{e}-T_{e}\right)-w_{\omega}
$$

the error equation given by (27) becomes

$$
\dot{e}_{\omega}=-\frac{B}{J} e_{\omega}+\mathbf{L}_{\omega} \mathbf{w}_{\Psi}+w_{\omega}^{\prime}=-\frac{B}{J} e_{\omega}+\mathbf{F w}
$$

where $\mathbf{F}=\left(\mathbf{L}_{\omega} 1\right)$ and $\mathbf{w}=\left(\mathbf{w}_{\psi} w_{\omega}^{\prime}\right)^{T}$.

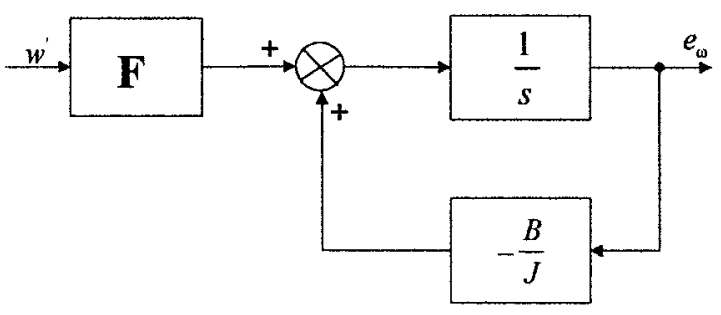

Fig. 4. Transfer function of error system.

For a realizable and stable system, the effect of disturbances and the convergence rate of errors can be analyzed by introducing two norms, $H_{2}$ norm and $H_{\infty}$ norm of the system transfer matrix, respectively. Through optimizing these norms under different operating conditions, an optimum switching gain can be obtained. In the Hardy spaces $H_{2}$ and $H_{\infty}$, the $\|T(s)\|_{2}$ is defined as

$$
\|T(s)\|_{2}=\frac{1}{2 \pi} \int_{-\infty}^{\infty} \operatorname{tr}\left[T^{*}(j \omega) T(j \omega)\right] d \omega
$$

where $j^{2}=-1$ and $T^{*}(j \omega)$ is a transpose and an adjoint matrix to the closed-loop transfer matrix of the error system $T(j \omega)$. Physically, $\|T(s)\|_{2}$ is the mean-square value of the estimation error corresponding to a stochastic noise and is also the integral of the square of the impulse response of the error system to the initial error. Therefore, the convergence rate of the error system can be quantitatively analyzed by using $\|T(s)\|_{2}$. On the other hand, the $\|T(s)\|_{\infty}$ norm is defined as

$$
\|T(s)\|_{\infty}=\sup _{\omega} \sigma_{\max }(T(j \omega))
$$

where $\sigma_{\max }[T(j \omega)]$ is the maximum singular number of the matrix $T(j \omega)$, which can be obtained by

$$
\sigma_{\max }[T(j \omega)]=\left[\lambda_{\max }\left(T^{*}(j \omega) T(j \omega)\right)\right]^{\frac{1}{2}}
$$

where $\lambda_{\max }$ is the maximum eigenvalue of the matrix $T^{*}(j \omega) T(j \omega)$. From (31), it can be seen that the $H_{\infty}$ norm represents the gain of $T(s)$ corresponding to the worst disturbances $\mathrm{w}$. Therefore, the $H_{\infty}$ norm, in practice, is the criteria of error system performance in a sense of robustness of disturbances. For a desired closed-loop system, the initial errors should be quickly attenuated, whereas the convergence rate of estimation errors can be evaluated by the $\mathrm{H}_{2}$ norm of the closed-loop system. The block diagram of error system $e_{\omega}$ is shown in Fig. 4, and the corresponding closed-loop transfer function is given by

$$
T_{\omega}(s)=\left(s+\frac{B}{J}\right)^{-1} .
$$

According to (30), the $H_{2}$ norm of (33) can be expressed as

$$
\left\|T_{\omega}\right\|_{2}=\frac{1}{2 \pi} \int_{-\infty}^{\infty} \operatorname{trace}\left[T_{\omega}(-j \omega)^{T} T_{\omega}(j \omega)\right] d \omega=\frac{J}{2 B} .
$$

From (34), the $\mathrm{H}_{2}$ norm of the closed-loop system is determined by the system parameters. Therefore, the convergence 
rate of initial errors is determined by the system inherent characteristics. From Fig. 4, the transfer matrix from disturbances $\mathbf{W}^{\prime}(s)$ to error output $\mathbf{E}_{\omega}(s)$ can be expressed as

$$
\mathbf{T}_{w e}(s)=\left(s+\frac{B}{J}\right)^{-1} \cdot \mathbf{F}
$$

where $\mathbf{F}$ is the disturbance input matrix defined by (29). From (31) and (32), the $\|\cdot\|_{\infty}$-norm of $\mathbf{T}_{w e}$ can be obtained as

$$
\begin{aligned}
\left\|\mathbf{T}_{w e}\right\|_{\infty} & =\sup _{\omega} \sigma_{\max }\left[\mathbf{T}_{w e}(j \omega)\right]=\left(\mathbf{L}_{\omega}^{2}+1\right)^{\frac{1}{2}} \cdot \frac{J}{B} \\
& =2 \cdot\|\mathbf{F}\|_{2} \cdot\left\|T_{\omega}\right\|_{2}
\end{aligned}
$$

where $\|\mathbf{F}\|_{2}=\left(\mathbf{L}_{\omega}^{2}+1\right)^{\frac{1}{2}}$ is the induced quadratic norm of $\mathbf{F}$. The $\left\|\mathbf{T}_{\text {we }}\right\|_{\infty}$ can be considered as the maximum gain of the error system. For the disturbances $\mathbf{W}^{\prime}(s)$, the maximum error response $E_{\omega \max }(s)$ is obtained as

$$
E_{\omega \max }=\left\|\mathbf{T}_{w e_{\omega}}\right\|_{\infty} \cdot \mathbf{W}^{\prime} .
$$

For any given constant $\alpha>0$ and disturbances $\mathbf{W}^{\prime}(s), E_{\omega \max }$ should be kept within the bounds of $\alpha$

$$
E_{\omega \max }=\frac{J}{B}\|\mathbf{F}\|_{2} \cdot\left\|\mathbf{W}^{\prime}\right\|_{2} \leq \alpha .
$$

According to the Cauchy-Schwarz inequality,

$$
|\langle\mathbf{F}, \mathbf{W}\rangle| \leq\|\mathbf{F}\|_{2} \cdot\|\mathbf{W}\|_{2}
$$

where $|\langle\mathbf{F}, \mathbf{W}\rangle|$ is the inner product, then (38) can be rewritten as

$$
\left[\left(\mathbf{L}_{\omega} \mathbf{w}_{\psi}\right)^{2}+\left(w_{\omega}^{\prime}\right)^{2}\right]^{\frac{1}{2}} \leq\left(\frac{\alpha B}{J}\right)
$$

From (40), an $H_{\infty}$ optimal observer can, therefore, be deduced under various system disturbances caused by parameter variations and load. Different situations are discussed as follows.

S1:

$$
\mathbf{r} \rightarrow(\mathbf{r}+\Delta \mathbf{r}): \mathbf{w}_{\psi}=-\Delta \mathbf{r} \mathbf{H}(\theta) \Psi
$$

and

$$
w_{\omega}^{\prime}=-\frac{\left(\hat{T}_{e}-T_{e}\right)+T_{1}}{J} .
$$

Thus, the inequality given by (40) can be written as

$$
\begin{aligned}
& {\left[\mathbf{L}_{\omega} \cdot(-\Delta \mathbf{r} \cdot \mathbf{H}(\theta) \Psi)\right]^{2}+\left(\frac{\left(\hat{T}_{e}-T_{e}\right)+T_{l}}{J}\right)^{2}} \\
& \quad \leq\left(\frac{\alpha B}{J}\right)^{2}
\end{aligned}
$$

By solving (41), an $H_{\infty}$ optimal $\mathbf{L}_{\omega}$ can be obtained as

$$
\begin{aligned}
& \left(\begin{array}{ll}
L_{\omega a} \quad L_{\omega b} \quad L_{\omega c}
\end{array}\right) \\
& \leq \frac{\left[(\alpha B)^{2}+\left(\hat{T}_{e}-T_{e}+T_{l}\right)^{2}\right]^{\frac{1}{2}}}{J} \\
& \quad \times\left(\frac{1}{\left|\Delta r_{a}\right| i_{a}} \frac{1}{\left|\Delta r_{b}\right| i_{b}} \frac{1}{\left|\Delta r_{c}\right| i_{c}}\right) .
\end{aligned}
$$

S2:

$$
\mathbf{H} \rightarrow(\mathbf{H}+\Delta \mathbf{H}): \mathbf{w}_{\psi}=-\mathbf{r} \Delta \mathbf{H}(\theta) \Psi
$$

and

$$
w_{\omega}^{\prime}=-\frac{\left(\hat{T}_{e}-T_{e}\right)+T_{l}}{J} .
$$

By substituting them into (40), it yields

$$
\begin{aligned}
& {\left[\mathbf{L}_{\omega} \cdot(-\mathrm{r} \cdot \Delta \mathbf{H}(\theta) \Psi)\right]^{2}+\left(\frac{\left(\hat{T}_{e}-T_{e}\right)+T_{l}}{J}\right)^{2}} \\
& \quad \leq\left(\frac{\alpha B}{J}\right)^{2}
\end{aligned}
$$

This inequality can be solved as

$$
\begin{aligned}
& \left(\begin{array}{ll}
L_{\omega a} \quad L_{\omega b} & L_{\omega c}
\end{array}\right) \\
& \leq \frac{\left[(\alpha B)^{2}-\left(\hat{T}_{e}-T_{e}+T\right)^{2}\right]^{\frac{1}{2}}}{J} \\
& \quad \cdot\left(\frac{1}{r_{a}\left|\Delta H_{a}\right| \psi_{a}} \frac{1}{r_{b}\left|\Delta H_{b}\right| \psi_{b}} \frac{1}{r_{c}\left|\Delta H_{c}\right| \psi_{c}}\right) .
\end{aligned}
$$

S3:

$$
B \rightarrow(B+\Delta B): w_{\omega}^{\prime}=-\frac{\left(\hat{T}_{e}-T_{e}\right)+T_{l}}{J}-\frac{\Delta B}{J} \omega
$$

and

$$
w_{\psi}=0 \text {. }
$$

The corresponding $H_{\infty}$ optimal $\mathbf{L}_{\omega}$ can be obtained as

$$
\left(-w_{\omega}^{\prime}\right)^{2} \leq\left(\frac{\alpha B}{J}\right)^{2}
$$

It should be noted that (45) is independent of $\mathbf{L}_{\omega}$, hence, it can be chosen arbitrarily large without violating the constraint of $H_{\infty}$. However, a very large $\mathbf{L}_{\omega}$ tends to give an undesirable dynamic error.

S4:

$$
\begin{gathered}
B \rightarrow(B+\Delta B) ; \quad \mathbf{H} \rightarrow(\mathbf{H}+\Delta \mathbf{H}) ; \quad(\mathbf{r}+\Delta \mathbf{r}): \\
\mathbf{w}_{\psi}=-(\Delta \mathbf{r} \mathbf{H}+\mathbf{r} \Delta \mathbf{H}+\Delta \mathbf{r} \cdot \Delta \mathbf{H}) \Psi
\end{gathered}
$$

and

$$
w_{\omega}^{\prime}=-\frac{\left(\hat{T}_{e}-T_{e}\right)+T_{l}}{J}-\frac{\Delta B}{J} \omega .
$$

Hence, (40) can be expressed as

$$
\begin{aligned}
& \mid L_{\omega a}\left(-\Delta r_{a} H_{a} \psi_{a}-r_{a} \Delta H_{a} \psi_{a}-\Delta r_{a} \Delta H_{a} \psi_{a}\right) \\
& +L_{\omega b}\left(-\Delta r_{b} H_{b} \psi_{b}-r_{b} \Delta H_{b} \psi_{b}-\Delta r_{b} \Delta H_{b} \psi_{b}\right) \\
& +L_{\omega c}\left(-\Delta r_{c} H_{c} \psi_{c}-r_{c} \Delta H_{c} \psi_{c}-\Delta r_{c} \Delta H_{c} \psi_{c}\right) \mid \\
& \quad \leq \frac{\left[(\alpha \beta)^{2}-\left(\hat{T}_{e}-T_{e}+T_{l}+\Delta B \omega\right)^{2}\right]^{\frac{1}{2}}}{J}
\end{aligned}
$$




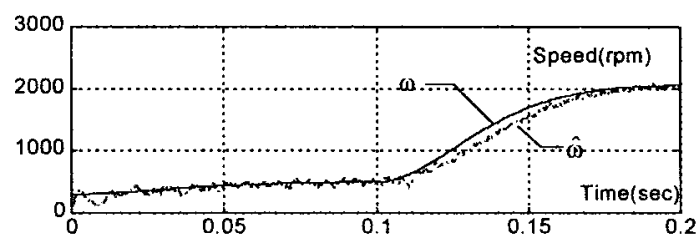

(a)

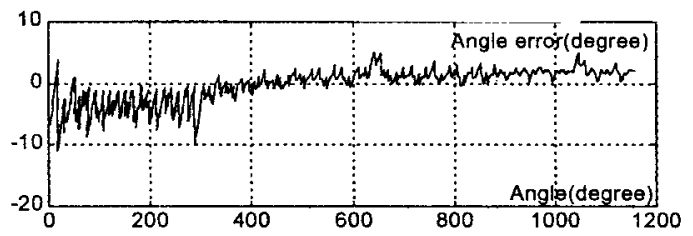

(b)

Fig. 5. Observer errors due to sudden change in reference speed. (a) Speed (speed versus time). (b) Position (angle error versus angle).

Solving (46), $\mathbf{L}_{\omega}$ can be obtained as

$$
\begin{aligned}
& \left(\begin{array}{lll}
L_{\omega a} & L_{\omega b} & L_{\omega c}
\end{array}\right) \\
& \leq \frac{\left[(\alpha B)^{2}-\left(\hat{T}_{e}-T_{e}+T_{l}+\Delta B \omega\right)^{2}\right]^{1 / 2}}{J} \\
& \cdot\left(\frac{1}{\left|\Delta r_{a}\right| H_{a} \psi_{a}+r_{a}\left|\Delta H_{a}\right| \psi_{a}+\left|\Delta r_{a}\right|\left|\Delta H_{a}\right| \psi_{a}},\right. \\
& \frac{1}{\left|\Delta r_{b}\right| H_{b} \psi_{b}+r_{b}\left|\Delta H_{b}\right| \psi_{b}+\left|\Delta r_{b}\right|\left|\Delta H_{b}\right| \psi_{b}}, \\
& \left.\frac{1}{\left|\Delta r_{c}\right| H_{c} \psi_{c}+r_{c}\left|\Delta H_{c}\right| \psi_{c}+\left|\Delta r_{c}\right|\left|\Delta H_{c}\right| \psi_{c}}\right) \text {. }
\end{aligned}
$$

Finally, $\mathbf{L}_{\omega}$ should be optimally determined to have a uniform ultimate value under the most severe situation among $\mathrm{S} 1, \mathrm{~S} 2, \mathrm{~S} 3$, and S4, without violating the inequality (40).

\section{COMPUTER SimUlation AND EXPERIMENTAL VERIFICATION}

\section{A. Computer Simulation}

For the sliding-mode observer given by (5), the optimal feedback gains $\mathbf{K}_{\psi}, \mathbf{K}_{\omega}$, and $\mathbf{K}_{\theta}$ can be determined by using (19), (23), (42), (44), and (47). The adequacy of the proposed control algorithm is testified by computer simulation based on a realistic three-phase 4-kW SRM drive. In the simulation, the following assumptions were made.

- $\hat{\Psi}(0)=0, \hat{\theta}(0)=0, \hat{\mathbf{i}}(0)=0, \mathbf{V}=\mathbf{V}_{s}$, and $\hat{\omega}(0)=$ $100 \mathrm{r} / \mathrm{min}$ are the initial conditions for overcoming the initial transients.

- Applied voltage and line current can be detected without time delay.

- Current-hysteresis-controlled PWM is employed.

Different simulations are performed under different initial states, speed references, load disturbances, and system parameter variations, so as to assess the robustness of the proposed sliding-mode observer. The simulation results are shown in Figs. 5-8.

Fig. 5 shows the observer errors of the estimated rotor position and motor speed subjected to a sudden change in

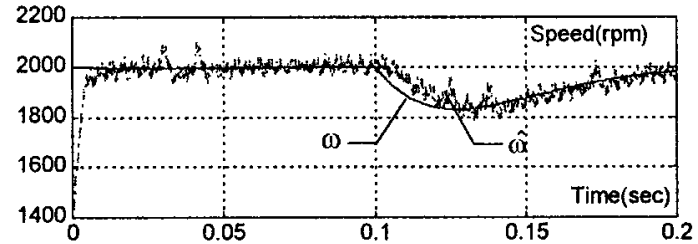

(a)

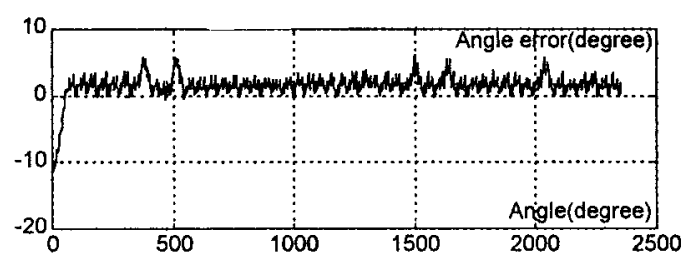

(b)

Fig. 6. Observer errors due to sudden change in load torque. (a) Speed (speed versus time). (b) Position (angle error versus angle).

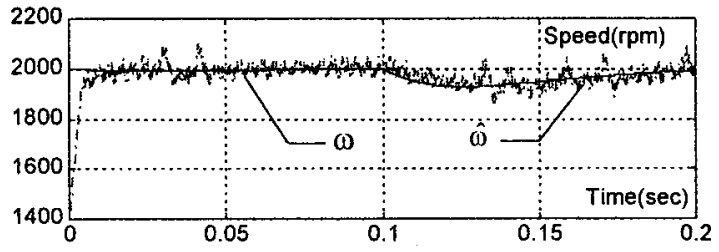

(a)

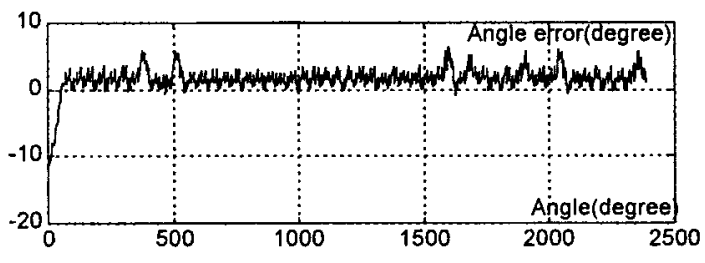

(b)

Fig. 7. Observer errors due to sudden change in damping coefficient. (a) Speed (speed versus time). (b) Position (angle error versus angle).

reference speed from 500 to $2000 \mathrm{r} / \mathrm{min}$ at $0.1 \mathrm{~s}$. The results indicate that the estimated positions can track the actual values, even under a large initial error. Figs. 6-8 illustrate the observer's errors due to various abruptly applied disturbances. The corresponding responses due to an abrupt step change of load torque from 5 to $13 \mathrm{~N} \cdot \mathrm{m}$ at $0.1 \mathrm{~s}$ are shown in Fig. 6. Although there are temporary increases in both speed and position errors, the system can quickly recover the expected tracking accuracy. Figs. 7 and 8 show the responses of the proposed observer in the presence of parameter and voltage variations, namely, an abrupt step- change of damping coefficient from 0.0012 to $0.0024 \mathrm{~N} \cdot \mathrm{m} \cdot \mathrm{s}^{2}$ at $0.1 \mathrm{~s}$ and an abrupt step change of supply voltage from 240 to $200 \mathrm{~V}$ at $0.1 \mathrm{~s}$. As expected, the observer can provide the insensitivity and robustness to these variations.

\section{B. Hardware Implementation}

For experimentation, a prototype of the proposed SRM drive is constructed. The system parameters of this three-phase 4-kW 


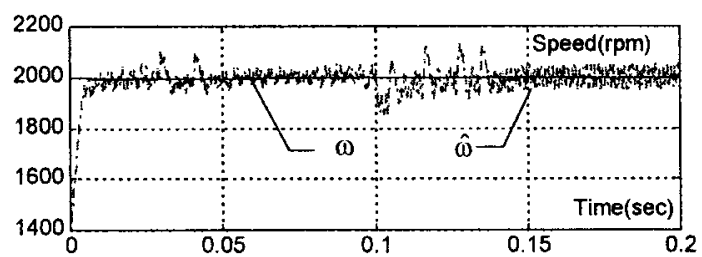

(a)

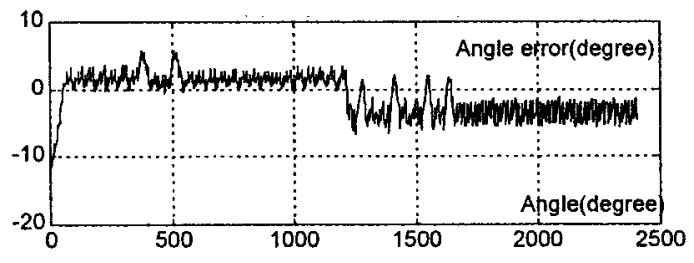

(b)

Fig. 8. Observer errors due to sudden change in supply voltage. (a) Speed (speed versus time). (b) Position (angle error versus angle).

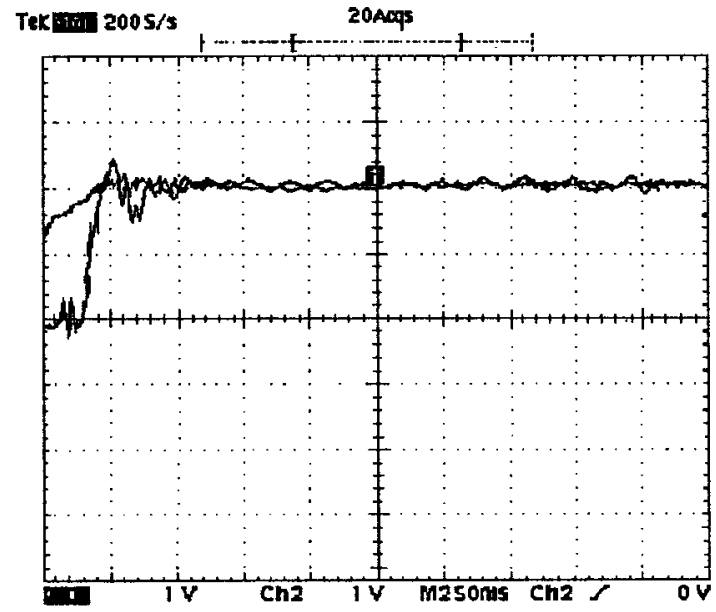

Fig. 9. Startup transient responses from 0 to $1200 \mathrm{r} / \mathrm{min}$ : (upper) measured speed; (lower) estimated speed; $600 \mathrm{r} / \mathrm{min} / \mathrm{div} ; 250 \mathrm{~ms} / \mathrm{div}$.

SRM drive are listed in Table I. The observer algorithm is implemented by the MC68HC16Z1 microcontroller, which can perform 32-bit floating-point multiplication, and can provide a machine cycle of only $150 \mathrm{~ns}$ for every computation. The sliding-mode observer runs on-line at a sampling rate of $50 \mu \mathrm{s}$. Moreover, the microcontroller deals with the phase current chopping and switching pattern computation for current and velocity control.

The SRM drive is first started up from an arbitrary initial rotor position $\theta_{0}$. When the starting procedure of about $150 \mathrm{~ms}$ is completed, the observer begins to work for the position and speed calculations. Fig. 9 shows the measured and estimated startup transient responses of the SRM drive, ranging from 0 to $1200 \mathrm{r} / \mathrm{min}$. It can be found that the proposed observer can quickly track the motor speed in less than $500 \mathrm{~ms}$. The corresponding voltage and current waveforms during $1200 \mathrm{r} / \mathrm{min}$ are also shown in Fig. 10, illustrating that the observer can provide accurate and stable rotor position estimation. Moreover, when there is a sudden change in reference speed from 500 to 1500

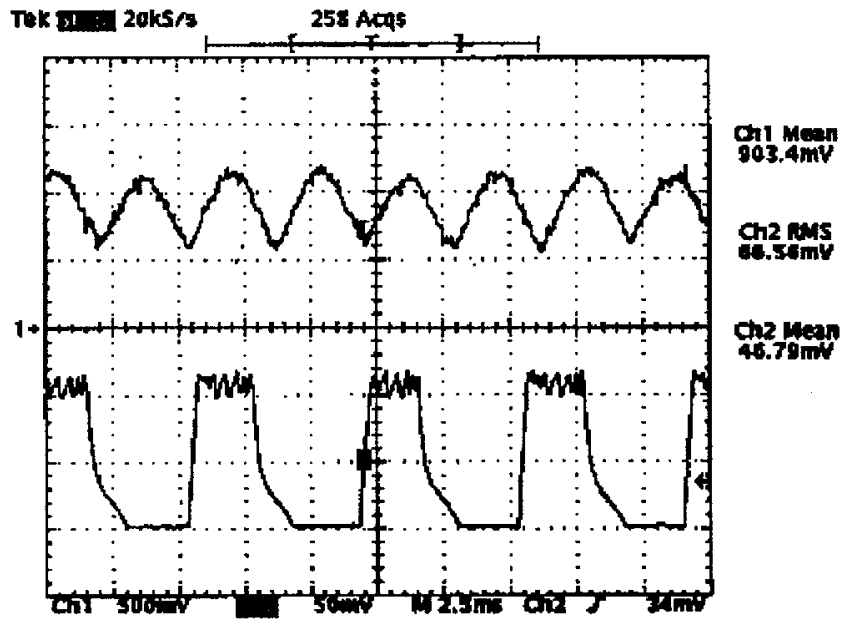

Fig. 10. Measured voltage and current waveforms at $1200 \mathrm{r} / \mathrm{min}$ : (upper) $125 \mathrm{~V} /$ div; (lower) $10 \mathrm{~A} / \mathrm{div} ; 2.5 \mathrm{~ms} / \mathrm{div}$.

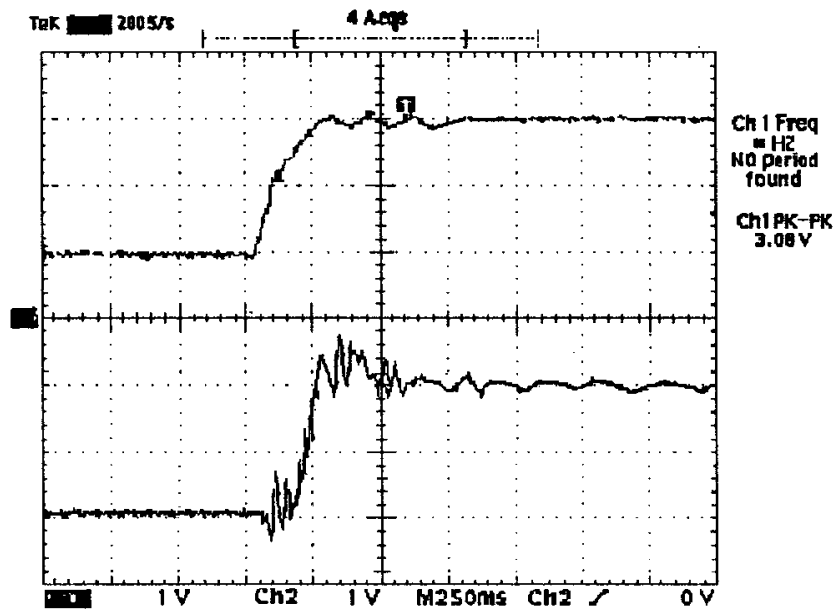

Fig. 11. Transient responses with sudden change in reference speed from 500 to $1500 \mathrm{r} / \mathrm{min}$ : (upper) measured speed; (lower) estimated speed; 500 $\mathrm{r} / \mathrm{min} / \mathrm{div} ; 250 \mathrm{~ms} / \mathrm{div}$.

$\mathrm{r} / \mathrm{min}$, the observer can quickly track the new speed in less than $500 \mathrm{~ms}$. The corresponding responses are shown in Fig. 11. Therefore, the practicability and performance of the proposed observer are verified.

\section{CONCLUSION}

A novel sliding-mode observer for an SRM drive has been proposed and implemented, in which magnetic saturation, load disturbances, parameter variations, and model errors have been taken into account. The stability of the proposed scheme is assured. The selection criteria of the corresponding switching gains are quantitatively analyzed to compensate for the system disturbances and parameter variations. Even starting the SRM drive under an arbitrary rotor position, the observer can provide a good transient performance. The feasibility, robustness, and convergence rate of the proposed observer are supported by the simulation and experimental results. 


\section{REFERENCES}

[1] C. Elmas and H. Z. Parra, "Position sensorless operation of a switched reluctance drive based on observer," in Proc. EPE Conf., 1993, pp. 82-87.

[2] M. Ehsani, S. Mahajan, K. R. Ramanim, and I. Husain, "New modulation encoding techniques for indirect rotor position sensing in switched reluctance motors," in Conf. Rec. IEEE-IAS Annu. Meeting, 1992, pp. 430-438.

[3] Y. Liao and C. Sun, "A novel position sensorless control scheme for doubly fed reluctance motor drives," IEEE Trans. Ind. Applicat., vol. 30, pp. 1210-1218, Sept./Oct. 1994.

[4] R. Lagerquist, I. Boldea, and T. J. E. Miller, "Sensorless control of the synchronous reluctance motor," in Conf. Rec. IEEE-IAS Annu. Meeting, 1993, pp. 427-436.

[5] F. Nozari, P. A. Mezs, A. L. Julian, C. Sun, and T. A. Lipo, "Sensorless synchronous motor drive for use on commercial transport airplanes," IEEE Trans. Ind. Applicat., vol. 31, pp. 850-859, July/Aug. 1995.

[6] S. R. MacMinn, W. J. Rzesos, P. M. Szczesny, and T. M. Jahns, "Application of sensor integration techniques to switched reluctance motor drives," in Conf. Rec. IEEE-IAS Annu. Meeting, 1988, pp. 584-588.

[7] A. Lumsdaine and J. H. Lang, "State observers for variable reluctance motors," IEEE Trans. Ind. Electron., vol. 37, pp. 133-142, Apr. 1990.

[8] M. Ehsani and I. Husain, "Rotor position sensing in switched reluctance motor drives by measuring mutually induced voltages," in Conf. Rec. IEEE-IAS Annu. Meeting, 1992, pp. 422-429.

[9] I. Husain, S. Sodhi, and M. Ehsani, "A sliding mode observer based controller for switched reluctance motor drives," in Conf. Rec. IEEE-IAS Annu. Meeting, 1994, pp. 635-643.

[10] J. J. Slotine, J. K. Hedrick, and E. A. Misawa, "On sliding observer for nonlinear systems," J. Dyn. Syst., Meas., Contr., vol. 109, no. 3, pp. 245-252, Sept. 1987.

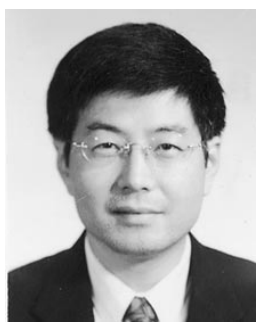

Y. J. Zhan (S'97) received the B.Sc. and M.Sc. degrees in electrical engineering from Hefei University of Technology, Hefei, China, in 1982 and 1986 , respectively. He is currently working towards the Ph.D. degree in the Department of Electrical and Electronic Engineering, The University of Hong Kong, Hong Kong.

His research interests include ac/dc motor design, drives, control theory and applications, power electronics, and technologies of electric vehicles.

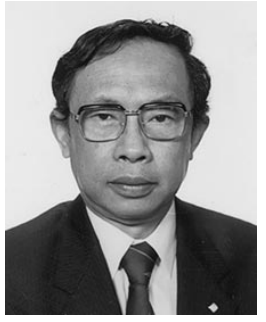

C. C. Chan (M'77-SM'77-F'92) received the B.Sc. degree from China University of Mining and Technology, Beijing, China, the M.Sc. degree from Tsing Hua University, Beijing, China, and the Ph.D. degree from The University of Hong Kong, Hong Kong, in 1953, 1957, and 1981 respectively, and was awarded the Honorary D.Sc. degree from the University of Odessa in 1993 for his prominent contribution to electric drives and electric vehicles.

$\mathrm{He}$ is currently the Endowed Honda Professor and Head of the Department of Electrical and Electronic Engineering, University of Hong Kong. His career includes extensive industrial and academic experience, mainly related to research and development of electric machines and power systems. He has been a Visiting Professor at many prominent universities around the world, including the University of California at Berkeley and Massachusetts Institute of Technology. He has a close collaboration with power utilities, industry, and governments as an adviser. In 1986, he founded the International Research Center for Electric Vehicles at The University of Hong Kong and hosted the 10th International Electric Vehicle Symposium in 1990 in Hong Kong. He is the Co-Founder of the World Electric Vehicle Association and is known as one of the "Three Wise Men" in the international electric vehicle community. He has authored four books, published over 120 technical papers, and holds seven patents. $\mathrm{He}$ has been extremely active in professional societies, especially the IEEE and HKIE, for over 20 years.

Prof. Chan is a Fellow of the Royal Academy of Engineering, U.K., a First Fellow of the Chinese Academy of Engineering in Hong Kong, and a Fellow of the Ukraine Academy of Engineering Science.

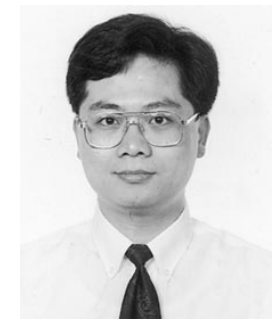

K. T. Chau (M'89) received the first-class honors B.Sc.(Eng.), M.Phil., and Ph.D. degrees, all in electrical and electronic engineering, from The University of Hong Kong, Hong Kong, in 1988, 1991, and 1993, respectively.

From 1990 to 1994, he was a Lecturer in the Department of Electrical Engineering, Hong Kong Polytechnic University. Since 1995, he has been with the Department of Electrical and Electronic Engineering, The University of Hong Kong, where he is currently an Associate Professor. He is the Coordinator of the Electric Vehicle Technology Research Group and the International Research Center for Electric Vehicles at The University of Hong Kong. His research interests include advanced motor drives, electric vehicles, and power electronics. He has published over 100 refereed technical papers and several industrial reports in these areas. He has been active in the area of electric vehicles and served as a Committee Member of the International Electric Vehicle Symposium.

Dr. Chau was the Publicity Chair of the IEEE International Symposium on Power Electronics Circuits in 1994 Pand the Technical Program Chairman of the IEEE Workshop on Switch Mode Power Supplies in 1995. He is a member of the IEEE Industrial Electronics and Power Electronics Societies and is listed in Who's Who in Science and Engineering 1998-1999. 